RESEARCH

Journal of Critical Dietetics

ISSN 1923-1237

Vol 6, Issue 2

Copyright 2022

Toronto, ON

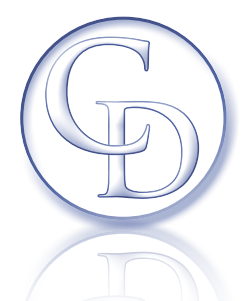

\title{
Analysis of the presentation of Nutritious Food Basket research on PHU websites in Ontario
}

Susan Belyea, PhD, Jennifer Brady, RD, PhD, Elaine Power, PhD

\begin{abstract}
The National Nutritious Food Basket (NNFB) is the only locally generated, standardized measure of the cost of a healthy diet gathered annually in Ontario (Ontario Ministry of Health Promotion (OMHP), 20I0). This study analyzed how research using the Nutritious Food Basket (NFB) protocol is presented and mobilized on the websites of public health units (PHU) for health equity advocacy in Ontario. The authors developed a coding scheme which was used to conduct a quantitative content analysis of the sampled websites with respect to four categories: I) presentation and framing of NNFB results; 2) strategies for communication of NNFB results; 3) calls to action based on NNFB results; 4) ease of navigation to NNFB results. In total, 35 PHU websites were analyzed. Thirty-two out of 35 websites included some information about local NNFB research, while three had none. The ways in which Nutritious Food Basket research is included, framed, and leveraged for advocacy on public health unit websites varies widely within each analytical categories developed for this research. Despite fairly consistent messaging that defines food insecurity as an income-related issue alongside statements that food banks are inadequate solutions, a surprising number of PHUs urge the public to donate to or volunteer for local food banks and other food charities in response to food insecurity. In most cases, Ontario PHU websites are not used as effectively as they could be for sharing NNFB results or for promoting advocacy activities based on NNFB research.
\end{abstract}

\section{Introduction}

The cost and affordability of a healthy diet is one of the myriad factors that impacts the prevalence and severity of food insecurity, and by which the adequacy of social policies, namely income support programs and minimum wages, may be assessed. Hence, routine monitoring of the cost and affordability of a healthy diet is vital to understanding food insecurity, and to informing health equity advocacy to redress food insecurity and its root cause, poverty. Health equity advocacy is defined as "a population health strategy that emphasizes collective action to effect systemic change. It focuses on changing upstream factors related to the social determinants of health, and explicitly recognizes the importance of engaging in political processes to effect desired policy changes at organizational and system levels" (National Collaborating Centre for Determinants of Health, 2015, pg. 3). Health equity advocacy is an important function of public health, and is a recognized activity for public health dietitians (PHDs) in Canada, whose roles makes them particularly well-positioned to advocate on food security. Access to data that examines the cost and affordability of a healthy diet in the local communities in which PHDs are employed is fundamental to their ability to advocate effectively and strategically to address food insecurity.

In Canada, monitoring the cost and affordability of a healthy diet is carried out by various levels of government, non-governmental organizations, researchers, and other stakeholders using a standardized survey tool 
known as the National Nutritious Food Basket, which is overseen by Health Canada, a department of the federal government (NNFB; Government of Canada, 2020a). The NNFB comprises a list of 67 foods for which a per week amount is stipulated to make up a basic healthy diet that meets the nutritional and caloric requirements for reference individuals of various gender and age categories (Government of Canada, 2020b). The standardization and quantification of a healthy diet via the NNFB's defined list of food items and specified quantities enables the collection of a clearly documented, locally generated, uniform, and unambiguous measure of the cost and affordability of a healthy diet that meets the national dietary guidelines that are entrenched in public health policy. In addition, the NNFB is the only such tool that exists in Canada to guide methodical, accurate, and rigorous food costing data. Hence, despite several deficiencies that are discussed in the next section, the NNFB is an important advocacy tool that enables measuring the cost of a healthy diet, as defined and endorsed across the multiple levels of government, against the public policies that govern important components of the social safety net, including social assistance and the minimum wage, both of which are provincially mandated.

Although the NNFB is a standardized survey tool, how it is operationalized also varies by province. Hence, the research presented here focuses on the use of the NNFB by public health units' (PHUs) in the province of Ontario, Canada to measure the cost and affordability of food, and more specifically, for the use of that data for health equity advocacy. In Ontario, data collected using the NNFB is the only routinely and locally generated, standardized measure of the cost and affordability of a healthy diet (Ontario Ministry of Health Promotion (OMHP), 20l0). NNFB data from across Ontario consistently show that social assistance and full-time minimum wage employment are inadequate for many Ontario households to afford a basic healthy diet

I A public health unit is "an official health agency established by a group of urban and rural municipalities" that administer, at a local level, the various functions of public health including the monitoring of health risk indicators, including social and behavioural determinants of health, the distribution of communicable and chronic disease, screening and immunization services, health education, as well as advocacy aimed at redressing health inequities related to the social and structural determinants of health (Ministry of Health and Long-term Care, 202I; National Collaborating Centre for Determinants of Health, 2015). once other basic necessities, such as housing, are paid. Although NNFB data is well positioned for use in evidence-based health equity advocacy related to food insecurity and poverty (Power, Belyea, and Collins, 2018), little research has been published to illuminate how and to what extent NNFB data is actually used in this way in Canada (Johnson, Williams, and Gillis, 2015). All Ontario PHUs maintain a website that could be a launching point for health equity advocacy initiatives (Galer-Unti, 2010). Hence, PHU websites are one obvious means by which the findings of NNFB food costing may be communicated to the public and policymakers, and thereby leveraged in for advocacy on food insecurity and poverty.

Of note is one body of literature that assesses the role of the internet and social media in public health interventions aimed at individual behavioral change (Bennett and Glasgow, 2009; Galer-Unti, 2010). However, little attention has been paid to the role of public health websites in structural change, such as through the communication of health research results like those of the NNFB, the dissemination of advocacy information, or the circulation of calls to action on health equity issues. The research reported here aims to aid in filling that gap in the literature, and more importantly, to provide insight regarding the strategic communication and use of NNFB data to inform the strategies and messaging of advocacy initiatives. More specifically, this research assesses the extent to which PHUs, in Ontario, have leveraged NNFB data via their websites.

\section{The National Nutritious Food Basket}

A prefatory version of the national nutritious food basket was first developed by the Food Prices Review Board (FPRB) in 1974 in response to public concern regarding what the FPRB describes in its 61 st and ultimate report titled, Telling It Like It Is, as the "1973-1976 Food Price Surge" (FPRB, 1976, pg. 37). It was not until 1997 that Health Canada took over responsibility for the NNFB, which has since included updates in 1998, 2008, and 2019 to align with emerging nutrition knowledge, changing food consumption patterns, and updates of other nutrition-related federal policy documents, such as Canada's Food Guide (Government of Canada, 2020a). Although Health Canada, a department of Canada's federal government, maintains the NNFB, Health Canada does not use the NNFB to collect such data, nor is the collection of food costing data using the NNFB included in the mandates of any level, department, or 
office of government in Canada. Although this obvious gap in public policy weakens the regard of the NNFB and the results of NNFB food costing data, including the resources made available for advocacy by PHUs and other stakeholders, this is not a weakness of the NNFB as a data collection or advocacy tool.

The twenty-two reference age and gender categories and the variety and stipulated quantities of each food included in the most recent NNFB, which was released in 2019, reflect the most recent version of the Dietary Reference Intakes (DRIs), nutrient data of foods gathered from the Canadian Nutrient File, and data on Canadians' consumption patterns acquired from the 2015 cycle of the Canadian Community Health Survey, a biannual, nationally representative health survey that tracks longitudinal patterns of various health indicators among Canadians. Despite being informed by a robust array of data, the NNFB survey tool has various epistemological and methodological strengths and weaknesses that are relevant to understanding the opportunities and limitations of NNFB food costing data for use in communications and advocacy.

First, it is important to note that although the NNFB outlines detailed diets for the twenty-two reference individuals included in the DRIs (i.e. body/girl aged 4-8; female/male aged 19-30, pregnancy or lactation at various ages), the NNFB is not meant to be definitive nor prescriptive of healthy eating at an individual level. From an epistemological perspective, the NNFB may be seen as promulgating what Hayes-Conroy and Hayes-Conroy (20II) name "hegemonic nutrition" (pg. I). Hegemonic nutrition comprises an array of problematic ways of conceptualizing, such as standardization, quantification, and presumed universality, that are rooted in positivist epistemology. However, we assert that it is also vital for those critical of hegemonic nutrition, and by extension the NNFB, not throw the baby out with the bath water and dismiss the utility of the NNFB as an advocacy tool, which hinges on its means to quantify the cost of a basic healthy diet that meets the recommendations of the same governments that are providing criminally low rates of income support for individuals and families living in poverty, as well as failing to implement policies such as a livable minimum wage and proven anti-poverty measures such as Basic Income.

From a methodological perspective, the NNFB bears a number of limitations that must be considered in interpreting and using results in advocacy initiatives. One major limitation lies in the various items that are not accounted for by the NNFB. For example, the narrow array of foods included in the 67-item list of the NNFB does not include: foods required to manage disease conditions (i.e. diabetes, heart disease, celiac disease) or allergies and intolerances; culturally diverse foods that are often supplied by niche producers or imported to Canada; foods necessary for religious or spiritual reasons (i.e. halal, kosher); and infant formulas. Foods required for any of the above reasons tend to be particularly costly, and would significantly increase the cost and affordability of food for individuals and families. Moreover, the cost of a food item included in the NNFB list typically reflects the least costly means of accessing that food at the time of data collection. Hence, it is the cost, not the convenience, accessibility, or any other factor that might determine how, why, and where individuals and families actually purchase food that determines the price for a food item that is recorded in the data collection process. However, an additional 5\% is added to the total of the cost of food per reference individual to account for items that are infrequently purchased, such as seasonings and cooking oils.

Another methodological limitation of the way that the NNFB is used, but not necessarily of the NNFB itself, is that reliable inter-provincial and inter-territorial comparisons are not possible because different protocols are used to operationalize data collection within each province and territory of Canada (i.e. time of year data is collected, type and variety of food retailers from which data is collected). Despite these limitations, the NNFB remains important as one tool that enables the standardized, and localized measure and longitudinal monitoring of the cost and affordability of a healthy diet that meets the basic needs of most Canadians. However, the NNFB enables longitudinal and localized (i.e. within towns, cities, rural regions) comparisons of the cost and affordability of food within the provinces and territories of Canada. Although the inability to make inter-provincial and inter-territorial comparisons is a limitation of how the NNFB tool is used across Canada, existing social policies to redress food insecurity and poverty, such as social assistance and minimum wage, are administrated at the provincial and territorial level in Canada, which means that NNFB food costing data remains relevant in advocating for policy change. In Ontario, PHUs have played a central role in leading the collection of food costing data using the NNFB, but given gaps in the literature, it remains unclear how, and the extent to which, PHUs have leveraged NNFB food costing data to inform health equity advocacy. 


\section{Purpose}

The overarching purpose of this research is to assess the extent to which NNFB food costing data is mobilized via the websites of Ontario-based PHUs for health equity advocacy initiatives. To conduct this assessment, this research, more specifically aimed to analyze if and how NNFB research is presented on the websites of Ontario PHUs.

\section{Method}

The content of the websites of all 35 public health units in Ontario was surveyed in July and August of 2018. The PHU websites examined for this research were identified from the website of the Ontario Ministry of Health and Long-Term Care (Ontario Ministry of Health and Long-term Care (OMLTC), 20I0). After identifying the website URLs, an inductive process was used to develop a coding scheme based on an initial review of each website. The inductive process used to develop the coding scheme proceeded as follows: Each PHU website was scanned for mentions of the NFBB. All mentions of the NFBB found on PHU websites were entered into Atlas.ti (Scientific Software Development $\mathrm{GmbH}, 2021$ ) qualitative data analysis software. Patterns were observed regarding how NFBB content was included on PHU websites, which resulted in the development of the coding scheme, which comprises four categories of analysis. The categories of analysis include: I) presentation and framing of NNFB results; 2) strategies for communication of NNFB results; 3 ) calls to action for advocacy based on NNFB results; 4) ease

Table I Categories, codes, and description of codes used for analysis of PHU websites

\section{Categories}

Presentation and research
Framing of NNFB

\section{Codes}

NFB Reporting

Analysis and framing of dimensions of food insecurity

\begin{tabular}{|c|c|c|}
\hline \multirow[t]{3}{*}{$\begin{array}{l}\text { Strategies for } \\
\text { Communication }\end{array}$} & Tools: Infographics or videos & $\begin{array}{l}\text { Are there communication tools such as infographics or } \\
\text { videos that communicate key elements of NNFB research? }\end{array}$ \\
\hline & $\begin{array}{l}\text { Evidence of media release about } \\
\text { NNFB }\end{array}$ & $\begin{array}{l}\text { Is there evidence that a media release had been issued } \\
\text { about NNFB findings within the past two years? }\end{array}$ \\
\hline & $\begin{array}{l}\text { Evidence of using NNFB data in } \\
\text { community coalition on poverty and/ } \\
\text { or food security }\end{array}$ & $\begin{array}{l}\text { Is there evidence that NNFB data was shared with } \\
\text { community partners working on issues of food security } \\
\text { and/or poverty? }\end{array}$ \\
\hline \multirow[t]{8}{*}{ Calls to Action } & $\begin{array}{l}\text { Link to the No Money for Food is } \\
\text { Cent\$Less Campaign }\end{array}$ & $\begin{array}{l}\text { Is there a link to the "No Money for food is фentsless" } \\
\text { campaign endorsed by ODPH? }\end{array}$ \\
\hline & $\begin{array}{l}\text { Advocacy recommendations to } \\
\text { Board of Health }\end{array}$ & $\begin{array}{l}\text { Do PHD reports to Board of Health recommend } \\
\text { advocacy action based on NNFB research? }\end{array}$ \\
\hline & $\begin{array}{l}\text { Call to Action: advocate for income } \\
\text { solutions to food insecurity }\end{array}$ & $\begin{array}{l}\text { Is there a clear call for the public to advocate for income } \\
\text { based solutions to food insecurity? }\end{array}$ \\
\hline & $\begin{array}{l}\text { Call to Action: Educate yourself and } \\
\text { others about food insecurity }\end{array}$ & $\begin{array}{l}\text { Is there a clear call for the public to self-educate and } \\
\text { educate others about food insecurity in the region? }\end{array}$ \\
\hline & $\begin{array}{l}\text { Call to Action: Share information } \\
\text { about food insecurity on social media }\end{array}$ & $\begin{array}{l}\text { Is there a clear call for the public to share information } \\
\text { about the NNFB from the PHU website on social media }\end{array}$ \\
\hline & Call to Action: Contact politicians & $\begin{array}{l}\text { Is there a clear call for the public to contact politicians } \\
\text { regarding policy change for food insecurity }\end{array}$ \\
\hline & $\begin{array}{l}\text { Call to Action: Volunteer or donate } \\
\text { to a charitable food program. }\end{array}$ & $\begin{array}{l}\text { Is there a clear call to the public to address food insecurity } \\
\text { by volunteering and/or donating to food banks and other } \\
\text { food programs? }\end{array}$ \\
\hline & Call to Action: Other & $\begin{array}{l}\text { Other suggestions for action on food insecurity including } \\
\text { buy local food; participate in food policy groups; etc. }\end{array}$ \\
\hline $\begin{array}{l}\text { Ease of Navigation } \\
\text { to NNFB data }\end{array}$ & $0-4$ & $\begin{array}{l}\text { Assigned numerical value based on ease of navigation to } \\
\text { NNFB findings on website }\end{array}$ \\
\hline
\end{tabular}

Description of code

Is a full NNFB report available for download from the website?

Is there a clear explanation of food insecurity as an income-related issue that requires income-based solutions?

Are there communication tools such as infographics or videos that communicate key elements of NNFB research? Is there evidence that NNFB data was shared with community partners working on issues of food security poverty?

Is there a link to the "No Money for food is фentsless" NNFB findings on website 
of navigation to NNFB results. The four categories of analysis were further developed by identifying indicators and corresponding descriptions that elaborated and operationalized each category for use in the analysis of the PHU websites (see Table I). Thus, the categories of analysis and corresponding indicators and descriptions constructed a unique coding scheme wherein the indicators served as codes that were applied in the process of conducting the analysis. To conduct the analysis, each website was thoroughly reviewed at least twice and analyzed using the unique coding scheme developed for this research.

\section{Results}

Thirty-two out of the 35 websites analyzed for this research included some information about local NNFB research. In the other three cases there was no information pertaining to NNFB research on the website. We provide a detailed report of our findings below, which is organized according to the coding scheme that we developed using the inductive process described above.

\section{Category I: Presentation and framing food insecurity}

This category comprises two indicators: I) NNFB Reporting; 2) Analysis and framing of dimensions of food insecurity. Results for both indicators associated with this category of analysis varied widely across the Ontario $\mathrm{PHU}$ websites analyzed for this research. NNFB results were found in the form of a downloadable document on $24(68.57 \%)$ of the $35 \mathrm{PHU}$ websites. Twenty one $(87.5 \%)$ of these offered a stand-alone, downloadable report on NNFB research, and three (12.5\%) PHU websites embedded their NNFB results within longer downloadable reports to the Board of Health. Eight websites presented information about the NNFB in non-downloadable narrative format on a webpage, or in the form of infographics that were not downloadable. Presenting NNFB data in the form of a downloadable report is advantageous to mobilizing the data because doing so means that users may more readily preserve, link to, share, and reference the downloadable document (See Table 2).

The 24 downloadable NNFB reports, including both standalone reports and results embedded in reports to boards of health, varied in length and detail. Five websites (20.83\%) did not have reports current at the time of data collection (2017 or 2018), but had reports from 2015 or 2016. Most reports were between four and six pages long and included data on different household scenarios and average housing costs, along with some explanation of the NNFB research protocol. These reports typically included a discussion of the cause of food insecurity, along with suggestions for policy responses and advocacy activities for the public. Some reports were as short as two pages, presenting only a brief explanation of how NNFB research is conducted and the calculated cost of feeding a reference family of four (a man and woman, each aged 31- 50 years; a boy, 14 to 18 years of age; and, a girl, 4 to 8 years old) in their jurisdiction (OMPH, 20I0). Short reports tended not to present multiple household scenarios. Some short reports were, however, augmented with other materials on food insecurity including infographics ( $n=20 ; 57.14 \%)$ or links to local anti-poverty or food security coalition documents ( $n=15 ; 42.85 \%)$ as discussed in more detail below.

Table 2. Summary of information available on PHU websites surveyed

\begin{tabular}{lcc} 
& $\mathbf{n}$ & $\%$ \\
\hline Ontario PHU websites surveyed & 35 & 100 \\
\hline $\begin{array}{l}\text { Provided downloadable, stand-alone } \\
\text { NNFB reports }\end{array}$ & 21 & 60 \\
\hline $\begin{array}{l}\text { Provided NNFB results embedded } \\
\text { in board of health report }\end{array}$ & 3 & 8.57 \\
\hline $\begin{array}{l}\text { Provided NNFB data on webpage } \\
\text { but no downloadable report. }\end{array}$ & 8 & 22.86 \\
\hline $\begin{array}{l}\text { No Evidence of NNFB research } \\
\text { on website }\end{array}$ & 3 & 8.6 \\
\hline
\end{tabular}

The majority of the PHU websites frame food insecurity as a symptom of inadequate income, which is in line with research evidence and widely accepted definitions. Each of the $24 \mathrm{PHU}$ websites that offered NNFB results in a downloadable report included a definition of food insecurity as inadequate or insecure access to food because of financial constraints. All of these 24 $\mathrm{PHU}$ websites note that food insecurity is a serious public health concern because of its relationship with poor nutrition and negative physical and mental health effects among adults and children. Twenty-one (87.5\%) of these 24 websites also note that food banks and other community food programs are inadequate responses to the root cause of food insecurity, which is inadequate income. It is important that NNFB research is presented alongside a clear and consistent definition of household food insecurity. A clear and consistent definition is vital to framing the problem and developing coordinated health equity advocacy efforts across governmental and non-governmental groups. 
All of the 21 downloadable NNFB reports included Canada Community Health Survey (CCHS) data about food insecurity for their region or included links to food insecurity research or policy papers such as those produced by PROOF, a policy-focused interdisciplinary research team investigating household food insecurity in Canada (PROOF, 20I8), and the Ontario Dietitians in Public Health (ODPH; ODPH, 2017). The inclusion of data from the CCHS, PROOF, and ODPH is important to framing NNFB research on PHU websites because these data serve to contextualize NNFB research in a broader understanding of household food insecurity . For example, the inclusion of such data makes clear the connection between inadequate income and the prevalence and severity of food insecurity, as well as the ways that the prevalence of food insecurity is inequitably distributed among individuals impacted by additional forms of oppression (i.e. racism, colonialism, sexism).

Among the PHU websites that do not offer a downloadable report, there is considerable variation in the amount of NNFB information presented and how it is analyzed and framed. Content ranged from a detailed synopsis of NNFB findings, to a one-page explanation of how to use the NNFB protocol to calculate the cost of eating for a household. In some cases, information about the NNFB was spread across two or more unlinked pages on a website. In three cases, there was no information about NNFB costing which eliminates an opportunity for those websites to contribute to health equity advocacy. In several other cases, the NNFB webpage also lists food charities and information on budgeting. Including such information is problematic because it frames food insecurity in ways that are contrary to what the extensive body of literature on food insecurity demonstrates-that food insecurity is a problem of inadequate income, and is thus, not a problem that may be solved with food charity or consumer education. As well, such inconsistencies in framing food insecurity may be confusing for people and organizations engaging in health equity advocacy based on NNFB data. Disparities in how PHUs in Ontario present and communicate NNFB findings support the findings of Brasolotto, Raphael, and Baldeo that public health units vary widely in their understanding and application of concepts like the social determinants of health in their activities (Brassolotto, Raphael, and Baldeo, 20I4), and may reflect tensions that have been acknowledged by PHUs between their own upstream and downstream responses to food insecurity (Power, Belyea, and Collins, 2018).
Making NNFB findings available in an in-depth, downloadable report available on the PHU website is a logical way to share this information with community organizations, politicians, and individuals who are engaged in health equity advocacy. Moreover, research regarding social activism by non-profit organization indicates that the interactivity of online content, such as the ability to share digital content, enhances engagement and the reach of campaigns (Seelig, Millette, Zhou, Huang, 2018). Hence, when NNFB data are not readily shareable, such as by downloading and forwarding materials on to others, the reach and impact of advocacy prompted by $\mathrm{PHU}$ may be limited.

\section{Category 2: Strategies for communication}

Three indicators were used to assess communication strategies on PHU websites: I) NNFB infographics and/ or videos; 2) recent media release about NNFB results; 3) links to community coalitions on poverty and/or food security.

There is ample evidence of PHU's intent to communicate NNFB costing research to the general public through the presentation of infographics, videos and media releases, although these strategies were not universally used on the PHU websites. Dorfman identifies social math as a communication tool for public health advocacy (Dorfman and Daffner Krasnow, 2014). Social math draws out and translates key data so that they are interesting and meaningful to an audience. A common way of presenting social math is through an infographic - a chart, diagram, or illustration that uses graphic elements to present information in a visually striking way. Infographics based on NNFB results were found on 20 (57.14\%) PHU websites. Infographics typically include household scenarios, housing costs, and suggestions for action. Most were in a downloadable format that could be printed or shared on social media. Five (14.29\%) PHU websites also included short videos about food insecurity on their NNFB webpages.

In conducting this research, we found media releases about local NNFB research on 14 (40\%) Ontario $\mathrm{PHU}$ websites. While the evidence of a media release about NNFB research on a PHU website indicates a commitment to communicate NNFB data to the public, the absence of media releases may either be because no media release was issued, or because there is no website archive of media releases. Cohen and Marshall (2017) caution that media advocacy is a distinct and complex theme within public health advocacy literature. Further 
research would need to be done to verify the degree to which a media release was picked up and shared among media outlets, as well as to analyze the key messages in media releases about NNFB research.

One way that public health dietitians (PHD) may communicate findings from NNFB research is through partnerships with community organizations (Brassolotto, Raphael, and Baldeo, 2014; McPherson, Ndumbe-Eyoh, Betker, Oickle, and Peroff-Johnston, 2016) The NCCDH notes that "(m)any advocacy initiatives are organized through coalitions which bring together organizations to amplify their political power, share resources, and coordinate strategies" (NCCDH, 2015). Fifteen (42.85\%) PHUs have webpages that link to, or make reference to, a community coalition. Of the $35 \mathrm{PHU}$ websites reviewed, 23 (65.7I\%) link solely to food security coalitions, 4 (11.43\%) link only to antipoverty coalitions, and 3 (8.57\%) link to both. Where a link was provided to a report produced by a community coalition on poverty or food security issues, we found that the messaging about food insecurity and NNFB results was consistent with the framing of food security as a function of inadequate income that was found on NNFB webpages. Many PHUs work in collaboration with community coalitions on poverty or food security as another means for sharing NNFB results with a broader audience.

\section{Category 3: Calls to action}

This category of analysis comprises five indicators that elaborate the calls to action included on Ontariobased PHU websites based on NNFB data. Three of the indicators are defined by the audience targeted by the calls to action: I) the public; 2) boards of health; 3) policy makers. Delineating the various target audiences is relevant to describing the various strategies used on PHU websites for health equity advocacy. Two additional indicators captured inclusion of two wellpublicized advocacy initiatives that were based on NNFB results on Ontario-based PHU websites. The first initiative was led in 2015 by ODPH, and featured two key documents: a position statement titled, Position Statement on Responses to Food Insecurity Endorsements; and a report Income-related Policy Recommendations to Address Food Insecurity. The core message of the report is that households living with low incomes cannot afford rent, bills, and food, and is significant to PHU advocacy because it calls on the public and policy makers to advocate for income based solutions to food insecurity.
The second initiative, the No Money for Food is Cent\$less campaign (heareafter the Cent\$less campaign), was developed by the Simcoe Muskoka District Health Unit and launched in 2016. The Cent\$less campaign includes web-based infographics and a video on food insecurity, and is significant to this category of analysis because it was developed specifically as an advocacy campaign, encouraging people to educate themselves and others, and to engage with politicians and policy makers on issues that affect income and food insecurity, and because of its wide impact. The Cent\$less campaign has since been endorsed by the ODPH, which hosts Cent\$less campaign materials on their website along with position papers on income solutions to food insecurity.

Fifteen (42.85\%) PHUs and/or boards of health have formally endorsed the ODPH position paper on food insecurity (ODPH, 2017). Only 8 (22.86\%) of Ontario $\mathrm{PHU}$ websites provide a direct link to the ODPH Cent\$less campaign or make reference to other ODPH materials on their NNFB webpage. However, these 8 $\mathrm{PHU}$ websites all presented an abbreviated version of the three policy recommendation areas outlined in ODPH documents, including advocating for a basic income, increasing social assistance rates, and revising employment standards to better protect workers in precarious employment. Given the wide variation in NNFB-related messaging among PHU websites, it is worth noting the uptake of ODPH language and framing in communicating advocacy activities based on NNFB research. This supports previous findings that ODPH is an important support for PHDs doing health equity advocacy (Power, Belyea, and Collins, 2018)

Calls to action were also unevenly spread across PHU websites. Reports housed on PHU websites often included recommendations for the local board of health to engage in advocacy activities. For example, in two cases, we found reports that made recommendations that the Board direct the public health unit to continue using the NNFB protocol to monitor the cost of food. In three cases recommendations were made to boards of health to communicate with provincial ministries to take action on poverty reduction and/or food insecurity monitoring. However, complete data on advocacy recommendations to local boards of health that were not documented on PHU websites is not available.

Twenty eight (80\%) of PHU websites incorporated specific calls for action arising from NNFB findings. These calls for action were typically under the heading 
'What can you do about food insecurity?' These suggestions appear on webpages, in NNFB reports, or within infographics. The most common call for action, appearing on 24 (68.57\%) of PHU websites, directs the public to advocate for income solutions to food insecurity. Most websites do not offer concrete suggestions about how to advocate for income solutions. Eleven (31\%) websites urge people to vote strategically, offer contact information for politicians, or direct people to add their names to specific campaigns for income solutions to address food insecurity. The $24 \mathrm{PHUs}$ that direct the public to advocate for income solutions overlap significantly, though not completely, with the 24 PHUs that provide a downloadable NNFB report and frame NNFB findings within a definition of food insecurity as inadequate or insecure access to food due to financial constraints.

Nineteen (54.29\%) PHU websites encourage people to educate themselves and others about food insecurity. Seven of these specifically suggest sharing information about food insecurity through social media, suggesting that $\mathrm{PHUs}$ recognize the potential for using social media for health equity advocacy. However, despite fairly consistent messaging that defines food insecurity as an income-related issue alongside statements that food banks are inadequate solutions, a surprising number of PHU calls for action include urging the public to donate to or volunteer for local food banks and other food charities in response to food insecurity. Twelve (34.29\%) websites suggested that people volunteer or donate to a charitable food program as a response to food insecurity. This suggestion seemed to contradict statements that appeared elsewhere on the websites about the inadequacy of charitable food solutions for addressing food insecurity, and recommend advocacy for income-based solutions. Yet, this finding aligns with previous research indicating that the dominant cultural imperative to simply feed the hungry is a substantial obstacle to the consideration of upstream, incomebased responses to food insecurity (Power, 2017).

Finally, there were several other suggestions for action in response to food insecurity. These included advocating for affordable housing ( $n=8 ; 22.85 \%$ ); advocating for changes to employment standards $(n=8 ; 22.86 \%$; buying local food ( $n=4 ; 11.43 \%)$; and engaging in Circles, a trademarked poverty reduction program active in several Ontario communities $(n=4 ; 11.43 \%)$. In these cases, there was no clearly articulated explanation of how these activities would address the problem of food insecurity.

\section{Category 4: Ease of navigation}

Researchers identify a broad range of health equity advocacy activities that may be carried out by PHUs (Cohen and Marshal, 2017; NCCDH, 2015; Raphael and Brassolotto, 2015). However, there is little analysis of the role of $\mathrm{PHU}$ websites in communicating these activities. While many PHU websites offered NNFB research reports, infographics, and a good analysis of food insecurity, this information was often difficult to locate.

For each website, an attempt was made to navigate from the PHU homepage to NNFB research by navigating through menu items that would likely be intuitive for anyone seeking that information. Website searches began with menu items that contained words or phrases such as Nutrition, Healthy Eating, Food and Family Food Security, and Food Resources. When those did not lead to NNFB research results, menu items containing Statistics and Data, Reports, Health Equity, and Poverty were tried. When NNFB research data was not locatable by navigating through these seemingly logical menu items, $\mathrm{PHU}$ websites' internal search engines were used to locate information related to the NNFB research. In cases where the internal search engine also failed to yield results, a Google search for information related to NNFB research in a particular PHU jurisdiction was used. Each website was coded using numbers from $0-4$ based on difficulty of navigation (see Table 3 ).

Table 3. Navigability of websites

\begin{tabular}{|c|c|c|}
\hline Code & Meaning of Code & Incidence \\
\hline 0 & $\begin{array}{l}\text { No evidence of NNFB research found } \\
\text { on the PHU website or through an } \\
\text { independent google search }\end{array}$ & $n=3$ \\
\hline 1 & $\begin{array}{l}\text { Evidence of NNFB research found } \\
\text { outside the PHU website through an } \\
\text { independent google search, though } \\
\text { not through internal search. }\end{array}$ & $n=3$ \\
\hline 2 & $\begin{array}{l}\text { NFB research only found internal } \\
\text { PHU website search engine. }\end{array}$ & $n=10$ \\
\hline 3 & $\begin{array}{l}\text { NFB research findable by navigating } \\
\text { through logical menu items from } \\
\text { PHU homepage }\end{array}$ & $n=19$ \\
\hline
\end{tabular}

In 19 (54.29\%) cases an NNFB webpage was successfully located through menu items found on a PHU homepage. The navigational path to NNFB research differed widely across PHU websites; paths varied considerably in the 
logic behind them and in the number of clicks needed to access the information. An example of a logical path found on one PHU website was Nutrition>Food Access> NNFB. Another was Your Health>Healthy Living > Food and Healthy Eating > No money for food is Cent $\$$ less $>$ NFB. In many cases, it was more difficult to find the report by navigating from the home page menu. In several cases, menu items such as Nutrition or Healthy Eating did not lead to a page with NNFB results. Instead, NNFB data were located under menu items such as Reports, Health Stats, Poverty, or Financial Assistance. In most cases, it took more than one attempt to successfully navigate to NNFB reports. For example, in one case a search began by navigating from Home> Health Topics \& Programs $>$ Food \& Healthy Eating. When this did not lead to NNFB information Home> Health Topics \& Programs $>$ Health Equity $>$ Health equity resources. This, too was unsuccessful so Home $>$ Resources $>$ Research \& Statistics >Demographics \& Health Statistics. Finally, Home $>$ Resources $>$ Research \& Statistics $>$ Research \& Evaluation $>$ Reports and Knowledge Products was tried and led to the NNFB results sought.

In ten $(28.57 \%)$ cases NNFB results could not be located by navigating through logical menu items on the $\mathrm{PHU}$ homepage, but it was found using the PHU website's internal search engine. Successful search terms included Nutritious Food Basket, Cost of Eating, and Food Insecurity, although not all terms yielded successful searches in all cases. It typically took multiple attempts to find NNFB reports using internal search engines.

In three (8.57\%) jurisdictions, no evidence of NNFB data on the PHU website could be found either by navigating through the menu or by using the internal search engine, however the data was located by conducting an external (Google) search using the terms 'cost of eating' + '[jurisdiction]'. In two of these cases, the Google search led to a report made to a Board of Health that contained NNFB results. In the other case the search led to newspaper articles that referred to the research, indicating that research had been carried out and that a media release had been issued. Finally, in three cases, no publicly accessible evidence of NNFB research on the PHU website could be found, and was not locatable through an external web search.

\section{Conclusion}

This research provides a unique examination of how NNFB research is presented and can be mobilized for health equity advocacy through PHU websites in Ontario. While PHUs may use other mechanisms for communicating and promoting health equity advocacy based on NNFB findings, this research finds that most PHUs do not effectively use their websites for this purpose. It is apparent that PHD's in Ontario are committed to the role that NNFB data can play in understanding and addressing income related health inequities (Power, Belyea, and Collins, 2018). While PHU dietitians may have other ways of mobilizing and communicating NNFB results, in this era of digital advocacy, public health websites could be used more effectively for sharing NNFB data with the public, and for promoting health equity using the NNFB research. This research may aid PHDs by providing a means of sharing how NFBB research is mobilized via the websites of other PHUs, and by providing material for reflection regarding how to better feature the work they are engaged in to redress food insecurity.

\section{Limitations}

The main limitation of this research is the narrow scope of analysis used to evaluate how $\mathrm{PHUs}$ present, frame, and use the results of NNFB data for health equity advocacy. More specifically, our analysis does not capture how, if at all, PHUs may be using NNFB data for advocacy beyond their websites, namely in social media. Social media has become particularly important to advocacy compared to traditional forms of online media, such as websites, because of its potential to facilitate greater and speedier reach, engagement, and interactivity among stakeholders via sharing and user generated content (Seelig, Millette, Zhou, \& Huang, 2019). Future research should evaluate how, if at all, PHUs are using social media to leverage NNFB data for health equity advocacy, and what impact such strategies may have on mobilizing stakeholders to engage in change making.

\section{Implications for Practice}

While health equity advocacy is a recognized activity for public health dietitians in Canada, to the best of our knowledge there are no policies governing how PHUs share public health research or promote health equity advocacy based on the research that they carry out. PHDs spend considerable time on NNFB costing, and PHUs put significant resources into developing and maintaining their websites as public education tools. Based on this research, we make the following recommendations for PHUs and PHDs to better mobilize NFBB data, and thus, to make better use of the resources invested in 
NFBB food costing work:

- PHUs should ensure that NFBB data and results are included within and are easily accessible via their websites

- PHUs should accompany NFBB data with consistent and easily accessible messaging about food insecurity and health equity that highlights the structural causes of food insecurity, namely its connection to poverty

- PHDs could work more closely with PHU website developers to create easier search paths, consistent messaging, and clear calls to action for health equity advocacy based on local NNFB research

- PHUs should develop policy to govern how public health research, including but not limited to NFBB data, is accessible to the public and other stakeholders

\section{Author Bios:}

Susan Belyea completed a PhD in the School of Kinesiology and Health Studies at Queen's University in 2018. Her research focused on social and political responses to food insecurity in Canada and Latin America/the Caribbean. She is currently the director of the Ban Righ Centre at Queen's University, and is an adjunct professor in the department of Culture Studies. Susan continues to teach and lecture on issues of global and local food insecurity. She is active in community advocacy around poverty and food insecurity in Kingston, Ontario.

Jennifer Brady, PhD, RD, is Director and Assistant Professor in the School of Nutrition and Dietetics at Acadia University. Her work draws on mixed and qualitative methods to explore the intersections of food, health, and social justice, with an emphasis on health profession(al)s' roles in social justice, cultural food security, and the history and professionalization of dietetics.

Elaine Power, PhD is a Professor in the School of Kinesiology and Health Studies at Queen's University, where she teaches on topics such as food systems; critical weight studies; and qualitative research methods. She is the co-author of The Case for Basic Income: Freedom, Security, Justice.

\section{References}

Bennett, G. \& Glasgow, R. (2009). The delivery of public health interventions via the internet: Actualizing their potential. Annual Review of Public Health, 30, 273-292. doi:10.1146/ annurev.publhealth.031308.100235

Brassolotto, J., Raphael, D., and Baldeo, N. (20/4). Epistemological barriers to addressing the social determinants of health among public health professionals in Ontario, Canada: a qualitative inquiry. Critical Public Health, 24(3), 321-336. Doi:http://dx.doi.org/10.1080/09 581596.2013 .820256

Cohen, B.E. \& Marshal, S.G. (2017). Does public health advocacy seek to redress health inequities? A scoping review. Health and Social Care in the Community, 25(2):309328. Doi: http://doi: I0.11 II/hsc. 12320

Dorfman, L. \& Daffner Krasnow, I. (2014). Public Health and Media Advocacy. Annual Review of Public Health. 35, $293-$ 306. Doi: 10.1146/annurev-publhealth-032013-182503

Food Prices Review Board. (1976). Final Report: Telling it Like it is. http://oaresource.library.carleton.ca/wcl/2016/20161221/ RG27-10-1976-eng.pdf

Government of Canada (2020a, February 24). National Nutritious Food Basket. Health Canada. https://www. canada.ca/en/health-canada/services/food-nutrition/foodnutrition-surveillance/national-nutritious-food-basket.html

Government of Canada (2020b, February 24). The contents of the 2019 national nutritious food basket. Health Canada. https://www.canada.ca/en/health-canada/services/foodnutrition/food-nutrition-surveillance/national-nutritiousfood-basket/contents.html

Galer-Unti, R.A. (2010). Advocacy 2.0: Advocating in the digital age. Health Promotion Practice, II(6), 784-787. Doi:10.1177/1524839910386952

Hayes-Conroy, A. and Hayes-Conroy, J. (2013). Introduction. In Hayes-Conroy, A. and Hayes-Conroy, J. (Eds.). Doing Nutrition Differently: Critical Approaches to Diet and Dietary Intervention (pg. 23-40). New York: Ashgate Publishing.

Johnson, C., Williams, P.L., Gillis, D. (20I5). The capacity building experience of women engaged in determining the cost and affordability of healthy food in Nova Scotia, Canada. Journal of Hunger and Environmental Nutrition, I0(3), 356-378. Doi:10.1080/19320248.2014.962769

McPherson, C., Ndumbe-Eyoh, S., Betker, C., Oickle, D., \& Peroff-Johnston, N. (2016). Swimming against the tide: A Canadian qualitative study examining the implementation of a province-wide public health initiative to address health equity. International Journal of Health Equity. 15(I), 129. doi:http://doi.org/l0.1/86/s12939-016-0419-4

Ministry of Health and Long-term Care. (2021). Health Services in Your Community. Public Health Units. https:// www.health.gov.on.ca/en/common/system/services/phu/ 
National Collaborating Centre for Determinants of Health (2015). Let's Talk: Advocacy and Health Equity. Antigonish, NS: National Collaborating Centre for Determinants of Health, St. Francis Xavier University

Ontario Dietitians in Public Health. (2017). Position Statement on Responses to Food Insecurity Endorsements. https:// www.odph.ca/upload/membership/document/2017-04/ endorsement-list-3Imrl7.pdf

Ontario Dietitians in Public Health. (2015). Income-related Policy Recommendations to Address Food Insecurity. https://www.osnpph.on.ca/upload/membership/ document/recommendations-document-final.pdf

Ontario Ministry of Health and Long-term Care. (2018, Dec 21). Public Health Units. http://www.health.gov.on.ca/en/ common/system/services/phu/locations.aspx

Ontario Ministry of Health Promotion. (2010). The Nutritious Food Basket Guidance Document; Ontario. http://www.ontla. on.ca/library/repository/mon/24006/3020 I7.pdf

Power, E. (2017). Hunger and Poverty. In: P. Tortell, M. Young, \& P. Nemetz P. (Eds.), Reflections of Canada: Illuminating our opportunities and challenges at 150+ years (pp. 176-181). Peter Wall Institute for Advanced Studies.

Power, E.M., Belyea, S., and Collins, P. (2019). "It's not a food issue, it's an income issue": Using Nutritious Food Basket costing for health equity advocacy. Canadian Journal of Public Health, II0(3), 294-302. Doi: 10.17269/s41997-01900185-5

PROOF Food Insecurity Policy Research (2018, Dec 21). PROOF's research shows that food insecurity is a policy decision that requires income-based interventions to solve. https://proof.utoronto.ca

Raphael, D. \& Brassolotto, J. (2015). Understanding action on the social determinants of health: a critical realist analysis of in-depth interviews with staff of nine Ontario public health units. BMC Research Notes, 8(105). doi:http://DOII0.1186/ sl3104-015-1064-5

Scientific Software Development GmbH. (2021). Atlas.ti (Version No 22). https://atlasti.com/

Seelig, M.I., Millette, D., Zhou, C. \& Huang, J. (Catherine). (2019). A new culture of advocacy: An exploratory analysis of social activism on the web and social media, Atlantic Journal of Communication, 27(1), 15-29. doi: 10.1080/15456870.2019.1540418

Simcoe Muskoka District Health Unit. (2018). Basic Income pilot critical for reducing household food insecurity. http:// www.simcoemuskokahealth.org/HealthUnit/About/ Newsroom/NewsRelease/2017/Details/2017/04/25/ basic-income-pilot-critical-for-reducing-household-foodinsecurity 